\title{
A Research on the Application of MTI in Motion Parameters Measurement on Human-rifle System
}

\author{
Peng-Han GONG ${ }^{1,2, a}$, Ke-Dong ZHOU ${ }^{2, b}$, Hong HUANG ${ }^{3, \mathrm{c}}$, Xiao-Yong KANG ${ }^{2, \mathrm{~d}}$ \\ ${ }^{1}$ School of Mechanical Engineering, NUST, Nanjing 210094, China \\ ${ }^{2}$ The 1st Department, Ordnance Engineering College, Shijiazhuang 050003, China \\ ${ }^{3}$ School of Foreign Studies, NUST, Nanjing 210094,China

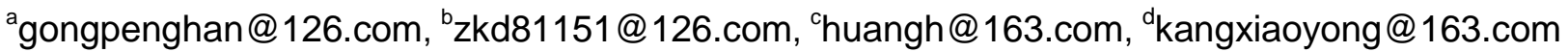

Keywords: MTI, Human-Rifle System, Motion Parameters, Attitude Measurement.

\begin{abstract}
This paper mainly focus on the principles and methods for measurement used in MTI (Motion Track Instrument) in an effort to study the feasibility of the inclusion of MTI in parameters measurement on human-rifle system. Focused on the fixation of MTI on the rifle and the motion features of human-rifle system during automatic firing, an analysis of the coordinate is carried out, based on which the combinational attitude algorithm of gyroscope, accelerometer and magnetometer are discussed. Through MTI, motion information on the angular velocity and the angular displacement at the muzzle of a three-shot burst rifle is collected. A comprehensive analysis on the muzzle response in pitching (up and down direction) and yawing (left and right direction) is then introduced. The findings show that the data acquired through MTI can precisely indicate the actual motion of human-rifle system, revealing that MTI is sufficient in measuring motion features of human-rifle system during automatic firing. Furthermore, they suggest MTI has great application potentials in motion parameters measurement and other fields.
\end{abstract}

\section{Introduction}

Motion parameters of human-rifle system during automatic firing is essential for dynamic modeling and analyzing motion features of human-rifle system. In accordance with measurement principle, there are two measurement methods of the motion parameters of human-rifle system, the non-contact measurement with optical equipment and the contact measurement with sensors[1]. The non-contact measurement with optical equipment requires many high-speed cameras working in a specific environment. The costs are rather high, yet it comes with limited measurement distance. In contrast, the contact measurement with sensors has fewer environmental demands and lower costs, thus it's widely employed in practice. According to Experimental research of man-gun movement influencing automatic firing accuracy(BAO Jiandong, WANG Chang-ming, KONG De-ren, et al) [2], researchers obtained the motion parameters of human-rifle system during firing through accelerometer array and gyroscope. However, this method requires a higher installation accuracy as well as the combined use of the instruments mentioned above, not to mention the involvement of a more complicated algorithm. In recent years, with the development of Micro Electron Mechanical System technology, the MEMS-based micro-inertial measurement system has been broadly applied to many fields, among which are aviation, biomedicine and military[3,4]. Taking the human-rifle system of a certain automatic rifle as the research subject, this paper focuses on the principle and the functions of MTI and its application in the measurement of human-rifle motion parameters.

\section{Brief Introduction of MTI}

MTI (Motion Track Instrument) is a MEMS-based micro-inertial measurement system, developed by XSENS Technologies in Netherlands. The system is composed of attitude sensors - enhanced gyroscope, accelerometer and magnetometer. The embedded processor which is interiorly integrated can calculate roll, pitch and yaw in real time, as well as collect calibrated 3D linear acceleration, angular velocity and earth's magnetic field data. 
MTI calculates the orientation between the sensor-fixed coordinate system and the earth-fixed coordinate system[5], both of which are shown in Fig.1.

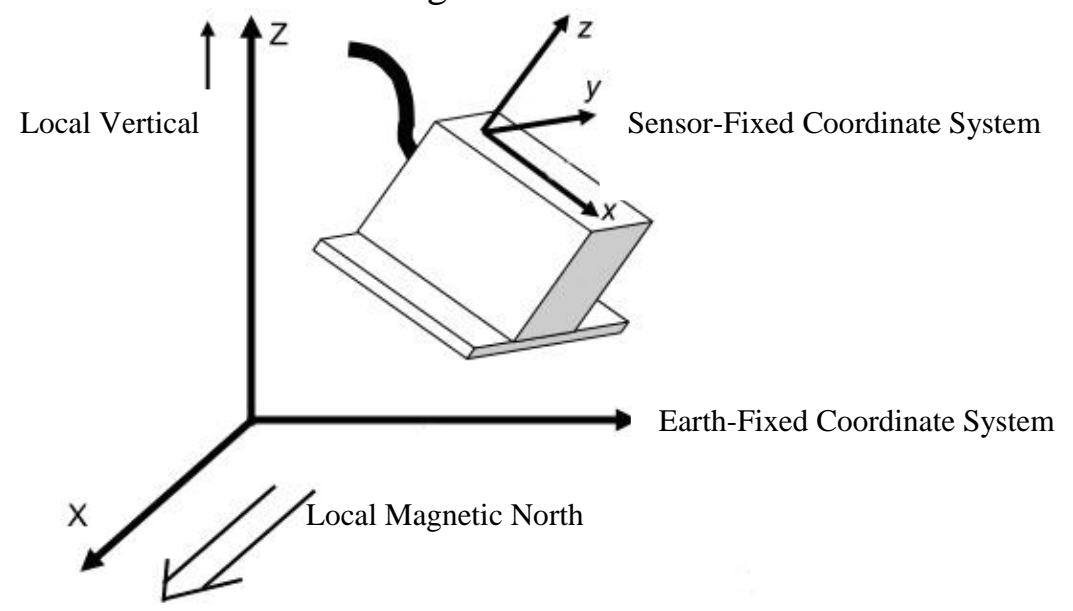

Fig.1 Sensor-Fixed Coordinate System and Earth-Fixed Coordinate System

\section{Mathematical Description on the Attitude Angle of Human-Rifle System}

The Attitude angle includes pitch, roll and yaw. The updating of attitude is the calculation of an attitude matrix, which processes the data of angular velocity collected in real time by gyroscope[6,7]. The quaternion orientation is usually used for the attitude updating. A quaternion is a matrix consisting of one real unit and three imaginary units $i, j, k$, which can be written in the following vector format:

$$
Q=\left(q_{0}, q_{1}, q_{2}, q_{3}\right)=q_{0}+q_{1} i+q_{2} j+q_{3} k=q_{0}+q
$$

In the formula, $q_{0}$ is a scalar, $q$ is a vector.

The Updating Equation of the quaternion in MTI system can be expressed as follows:

$$
\left(\begin{array}{l}
\dot{q}_{0} \\
\dot{q}_{1} \\
\dot{q}_{2} \\
\dot{q}_{3}
\end{array}\right)=\frac{1}{2}\left(\begin{array}{cccc}
0 & -\omega_{b x} & -\omega_{b y} & -\omega_{b z} \\
\omega_{b x} & 0 & \omega_{b z} & -\omega_{b y} \\
\omega_{b y} & -\omega_{b z} & 0 & \omega_{b x} \\
\omega_{b z} & \omega_{b y} & -\omega_{b x} & 0
\end{array}\right) \cdot\left(\begin{array}{c}
q_{0} \\
q_{1} \\
q_{2} \\
q_{3}
\end{array}\right)
$$

In the sensor-fixed coordinate system, $\omega_{b x}, \omega_{b y}, \omega_{b y}$ are the components developed by the resolution of angular velocity respectively along the $\mathrm{X}$ axis, $\mathrm{Y}$ axis and $\mathrm{Z}$ axis. The solution of this differential equation produces leads to four elements

$$
\left\{\begin{array}{l}
P_{1}=\frac{T}{2} q(t) \cdot \omega_{b}(t) \\
P_{2}=\frac{T}{2}\left[q(t)+\frac{P_{1}}{2}\right] \cdot \omega_{b}\left(t+\frac{T}{2}\right) \\
P_{3}=\frac{T}{2}\left[q(t)+\frac{P_{2}}{2}\right] \cdot \omega_{b}\left(t+\frac{T}{2}\right) \\
P_{3}=\frac{T}{2}\left[q(t)+P_{3}\right] \cdot \omega_{b}(t+T)
\end{array}\right.
$$


In the formula, $q(t), \omega_{b}(t)$ is the value at $t$ moment, and $T$ is the sampling period. Therefore, under given conditions, the quaternion value $q(t+T)$ at $t+T$ moment can be calculated. A rotation matrix can also be interpreted in terms of quaternions.

$$
\left[\begin{array}{ccc}
q_{1}^{2}+q_{0}^{2}-q_{3}^{2}-q_{2}^{2} & 2\left(q_{1} q_{2}+q_{0} q_{3}\right) & 2\left(q_{1} q_{3}-q_{0} q_{2}\right) \\
2\left(q_{1} q_{2}-q_{0} q_{3}\right) & q_{2}^{2}-q_{3}^{2}+q_{0}^{2}-q_{1}^{2} & 2\left(q_{2} q_{3}+q_{0} q_{1}\right) \\
2\left(q_{1} q_{3}+q_{0} q_{2}\right) & 2\left(q_{2} q_{3}-q_{0} q_{1}\right) & q_{3}^{2}-q_{2}^{2}-q_{1}^{2}+q_{0}^{2}
\end{array}\right]
$$

Substitute the four elements deduced above into (4). Through the relationship between quaternion and direction cosine, the attitude matrix can be solved and interpreted into data of yaw, pitch and roll.

$$
\left\{\begin{array}{l}
\theta=\sin ^{-1}\left(2 q_{0} q_{2}-2 q_{1} q_{3}\right) \\
\psi=\tan ^{-1}\left(\frac{2 q_{0} q_{3}+2 q_{1} q_{2}}{2 q_{0}^{2}+2 q_{1}^{2}-1}\right) \\
\phi=\tan ^{-1}\left(\frac{2 q_{0} q_{1}+2 q_{2} q_{3}}{2 q_{0}^{2}+2 q_{3}^{2}-1}\right)
\end{array}\right.
$$

\section{The Fixation of MTI on the Barrel}

After removing the stock, the percussion hammer, the bolt and breech block from the rifle, the gun was positioned on a platform, where a level calibration apparatus was used to make sure the barrel and the MTI remained strictly horizontal. Then, in order to ensure the X axis of the MTI is parallel to the barrel axis, a V-clamp made of aluminum alloy was designed and employed, which served to position the MTI on the end of the barrel. The fixation of the MTI on the barrel is shown in Fig.2.

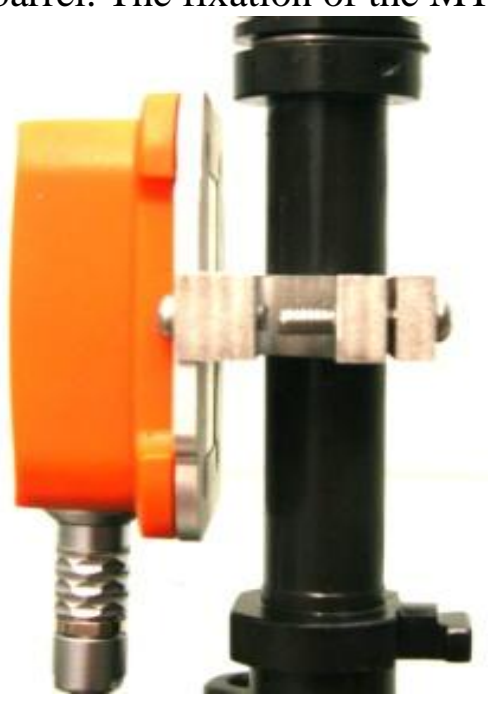

Fig.2 The Fixation of MTI on the Barrel

\section{Experiment and Result Analysis}

According to the curves of muzzle angular velocity shown in Fig.3 and 4, there was an obvious tendency of regular changes in the curves of angular velocity in pitching (up and down direction) and yawing(left and right direction). The results show that the angular velocity in pitching varied more 
than that in yawing, and they both achieved the maximum speed after the automatic machine had moved backward to position. The maximum angular velocity in pitching was $8.12 \mathrm{rad} / \mathrm{s}$, while that in yawing was $6.69 \mathrm{rad} / \mathrm{s}$. There was a strong correlation between the variation of angular velocity and the movement of the automatic mechanism, which can be useful in determining the influence the bore force and the automatic machine's moving forward and backward to position have on the angular velocity in pitching and yawing. There are $\mathrm{a}, \mathrm{b}$ and $\mathrm{c}$ three peaks on the time-angular velocity curve in pitching, as is shown in Figure 3. Peak a is caused by the bore force. Peak b is induced by the impact load as the bolt carrier hits the stock, while Peak c by the impact load as bolt carrier hits the receiver. The same correlation can be found in time-angular velocity curve describing each firing cycle under sustained firing, but it is less obvious. In particular, obvious discreteness was shown in the curve of angular velocity in yawing, which indicated randomness of the human-rifle system supported by human body.

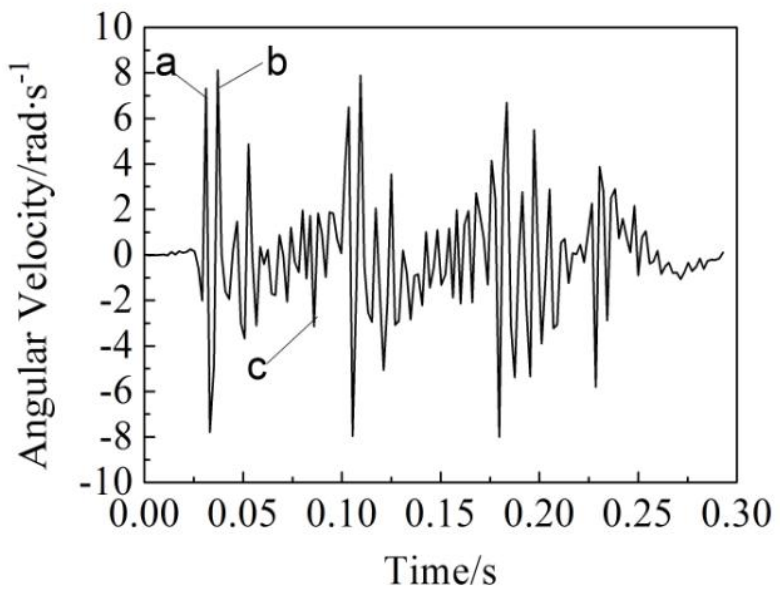

Fig.3 Angular Velocity of Muzzle in Pitching

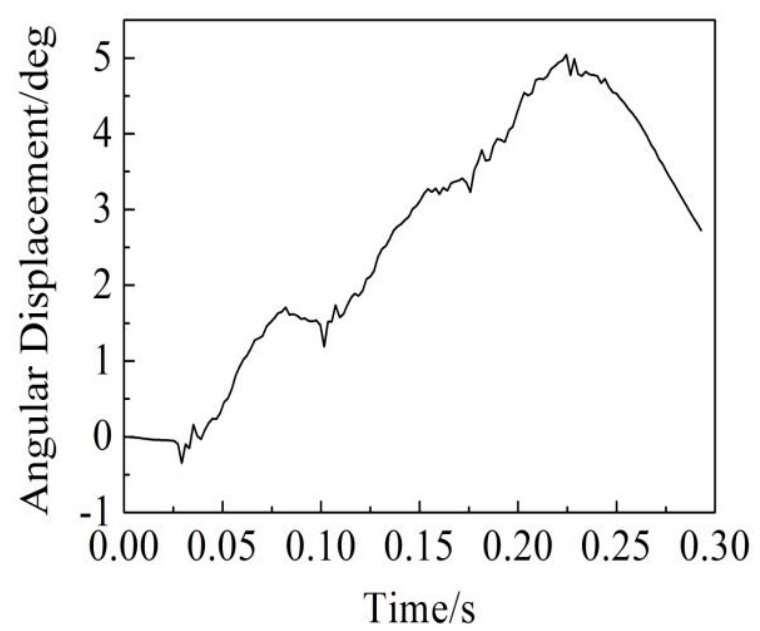

Fig.5 Angular Displacement of Muzzle in Pitching

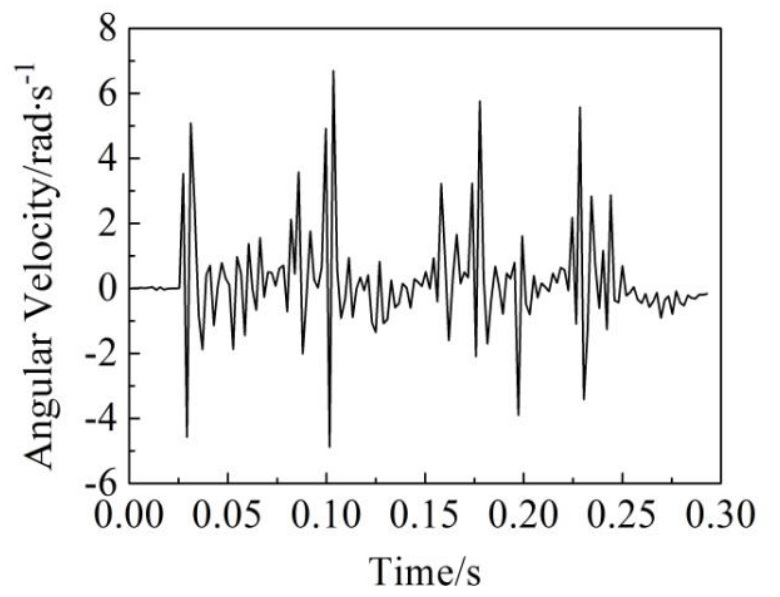

Fig.4 Angular Velocity of Muzzle in Yawing

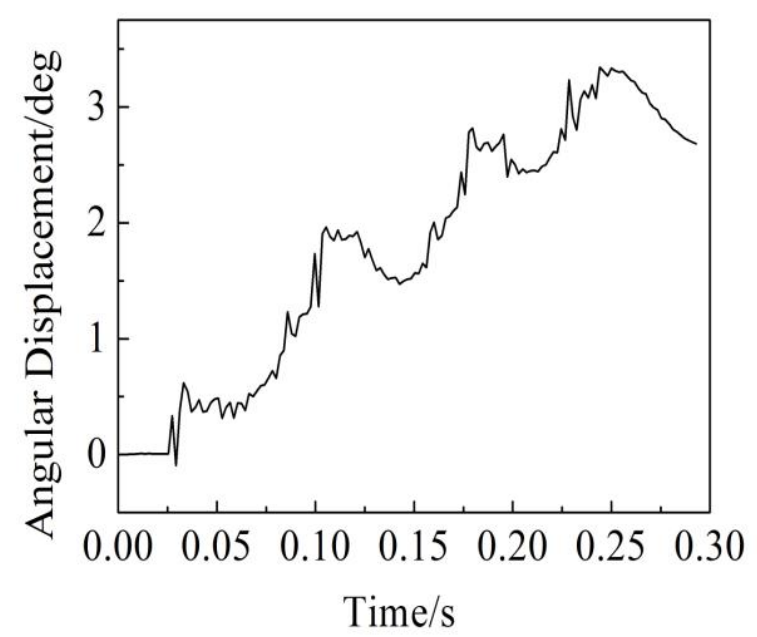

Fig.6 Angular Displacement of Muzzle in Yawing

The variation of angular displacement is shown in Figure 5 and 6 . The results show that angular displacement in pitching and yawing were gradually increasing. That is to say the movement of muzzle in pitching was always upward while that in yawing was always rightward, which was consistent with the bullet dispersion during automatic firing. There also exist corresponding points in angular displacement curve in accordance with the moment of the occurrence of bore force and automatic mechanism moving forward and backward to position. However, since these movements last very shortly, the corresponding angular displacements won't show much in the curve. Tab.1 shows the muzzle response as the bullet leaves the muzzle. 
Tab.1 Response of Muzzle as the Bullet Leaves the Muzzle

\begin{tabular}{ccccc}
\hline & \multicolumn{2}{c}{ Angular displacement (deg) } & \multicolumn{2}{c}{ Angular velocity (rad/s) } \\
\hline & pitching & yawing & pitching & yawing \\
\hline $1^{\text {st }}$ shot & 0.16 & 0.33 & 8.12 & 5.08 \\
\hline $2^{\text {st }}$ shot & 1.72 & 1.68 & 7.89 & 6.69 \\
\hline $3^{\text {st }}$ shot & 3.78 & 2.81 & 6.69 & 5.75
\end{tabular}

According to Tab. 1, during sustained firing the amplitude of muzzle response in pitching was greater than that in yawing, and the same pattern was found in bullet dispersion. In other words, muzzle response in pitching is the main factor determining the firing accuracy of a weapon.

\section{Conclusion}

Based on the comprehensive analysis of the principles and methods for measurement used in MTI, an MTI-oriented measurement method of motion parameters on human-rifle system was explored. The research findings show that MTI system with its good stability and good reproducibility can meet the measurement requirements of motion parameters on human-rifle system. The data collected in this paper are of great referential value to the study of motion law for human-rifle system and of the dynamic modeling of human-rifle system.

\section{References}

[1]Lee Young-Shin, Lee Se-Hoon, Choi Young-Jin, et al. Structural Human Impact Analysis on Shooting: Experimental and Simulation[J]. Key Engineering Materials, 2007(4): 3108-3111.

[2]BAO Jian-dong, WANG Chang-ming, KONG De-ren, et al. Experimental research of man-gun movement influencing automatic firing accuracy[J]. Chinese Journal of Scientific Instrument, 2006, 27(6):1274-1276.

[3]QIAO Yi-hui, CHENG Hui, MA Yan. MTi-based 3D Attitude Measurement[J]. Journal of test and measurement technology, 2011, (25)6:525-528.

[4]ZHANG Hai-peng, DANG You-yun. Human motion capture system based on MEMS sensors[J]. Journal of Xi' an Polytechnic University, 2012, 26 (1): 82-86.

[5]XSENS. MTI and MTX User Manual and Technical Documentation[Z]. //www. Xsense. com.

[6] LIU Jun, SHI Yun-bo, LI. Micro inertia Technologies [M]. Electrical Industry Publication, 20005:266-267.

[7]CHEN Le, ZHOU Jun-zhe. Research on MTI Miniature Posture Reference System Data Acquisition and Attitude Algorithm[J]. Journal of Shenyang ligong university, 2011, (30)4:31-35. 